\title{
An Entire Spectral Determinant for Semiclassical Quantization
}

\author{
Gábor Vattay \\ Niels Bohr Institute, Blegdamsvej 17, DK-2100 Copenhagen $\varnothing$, Denmark \\ and \\ Eötvös University, Múzeum krt. 6 - 8, H-1088 Budapest, Hungary
}

\begin{abstract}
We show that the eigenvalues of the first order partial differential equation derived by quasi-classical approximation of the Schrödinger equation can be computed from the trace of a classical operator. The derived trace formula is different from the Gutzwiller trace formula. The Fredholm determinant of the new operator is an entire function of the complex energy plane in contrast to the semi-classical spectral determinant derived from the Gutzwiller trace formula.
\end{abstract}

\section{Introduction}

The Gutzwiller trace formula [1] is the most important tool for semiclassical quantization of non-integrable systems. The trace formula, in general, does not converge close to the semiclassical energy eigenvalues or resonances where we would like to use it. The spectral determinant, derived by Voros 2 from the trace formula, has much better mathematical properties. However it also does not converge on the whole complex energy plane [3] and cannot be used to determine all the complex resonances of a system. One possible solution of the convergence problem is to introduce smeared trace formulas or spectral determinants. These methods limit the accuracy of the resonances and allows only qualitative comparison of the exact quantum and the semiclassical spectra. Trace formulas and spectral determinants can be introduced for classical systems [4 including thermodynamic formalism[5] and their analytical properties can be studied. The convergence problems of these formulas and the semiclassical convergence problems have the same origin [7]. For a large class of classical systems 
with 'nice' mathematical properties the recent theorem of H. H. Rugh[6] assures the convergence of classical spectral (Fredholm) determinants for a large class of classical systems with 'nice' mathematical properties. This theory can be extended to semiclassical trace formulas [8], and the semiclassical resonances can be computed without convergence problems. In addition, the numerical error of the semiclassical resonances decreases super-exponentially $\sim \exp \left(-n^{\beta}\right)(\beta>1)$ as a function of the maximal topological length $n$ of periodic orbits.

The theory in Ref. [8] has been developed on an abstract mathematical ground. Here, the connection of this mathematical description and the quasiclassical approximation, introduced by Maslov [10], is discussed. The term quasiclassical is more appropriate than semiclassical since the Maslov type description leads to a pure classical Perron-Frobenius operator in a natural way. The Fredholm determinant of this operator can serve as a well defined and mathematically clean starting point of the semiclassical approximation of the spectra. One additional advantage of this description is that the wave function evolves along one single classical trajectory and we do not have to compute sums over increasing numbers of classical trajectories as in computations involving Van Vleck formula 11.

\section{The quasi-classical approximation}

Following mostly Ref.[9], we give a summary of the quasi-classical approximation, which was worked out by Maslov[10] in this form. This approximation was used earlier by Liouville, Green, Stokes, Rayleigh and others.

The Schrödinger equation for a single particle of unit mass in a potential $U$ is

$$
i \hbar \frac{\partial \psi}{\partial t}=-\frac{\hbar^{2}}{2} \Delta \psi+U(q) \psi
$$

where $\Delta$ is the Laplace operator and $\psi(q, t)$ is the wave function. The $\hbar$ is the small parameter of the problem, and the quasi-classical approximation gives the solution of this equation for $\hbar \rightarrow 0$. This asymptotic solution is of short wave form

$$
\psi(q, t)=\varphi(q, t) e^{i S(q, t) / \hbar},
$$

where the amplitude $\varphi(q, t)$ and the phase $S(q, t)$ are smooth real functions on 
some bounded region of the configuration space. Substituting (2) in the Schrödinger equation, we can derive partial differential equations (PDE) for the amplitude and the phase with initial conditions $\varphi(q, 0)=\varphi_{0}(q)$ and $S(q, 0)=S_{0}(q)$ respectively. In the limit $\hbar \rightarrow 0$ omission of the non-leading terms proportional to $\hbar^{2}$ leads to the following quasi-classical partial differential equations

$$
\begin{aligned}
& \frac{\partial \varrho}{\partial t}+\operatorname{div}(\varrho \nabla S)=0, \\
& \frac{\partial S}{\partial t}+H(q, \nabla S)=0,
\end{aligned}
$$

where $\varrho(q, t)=\varphi^{2}(q, t)$ and $H(q, p)$ is the Hamilton function. Equation (四), which is the Hamilton-Jacobi equation, is a first order partial differential equation whose solution corresponding to an initial $S(q, 0)=S_{0}(q)$, can be given independently from (3). However, the continuity equation (3) can be solved only if the solution $S(q, t)$ of (4) is known. In other words, the Hamilton-Jacobi equation is autonomous while the continuity equation is driven by the solution $S(q, t)$ of (4).

We know from the theory of first order PDE's [12] that their solutions lead to ordinary differential equations (ODE). As is well known, the Hamilton-Jacobi equation leads to the Hamilton differential equations

$$
\begin{aligned}
\dot{q} & =\frac{\partial H(q, p)}{\partial p}, \\
\dot{p} & =-\frac{\partial H(q, p)}{\partial q},
\end{aligned}
$$

where the new variable

$$
p=\nabla S(q, t)
$$

has been introduced. In the PDE description we evolve the whole function $S(q, t)$ and compute its gradient at a given point $q_{0}$. Computation of the gradient requires information about the behavior of the function $S(q, t)$ in the vicinity of $q_{0}$ and can not be recovered from the value of $S\left(q_{0}, t\right)$ alone. In the ODE description we evolve both $q$ and $p=\nabla S(q, t)$. Therefore at a given time, we do not have to compute the gradient from $S(q, t)$ since the evolution is local in the $(q, p)$ space. From the ODE description we can reconstruct the whole solution of the PDE as

$$
S\left(q^{\prime}, t\right)=S(q, 0)+\int_{0}^{t} L(q(\tau), \dot{q}(\tau)) d \tau
$$


where we have to integrate the Lagrange function along the phase space trajectory with

$$
q^{\prime}=q(t), q=q(0), \nabla S\left(q^{\prime}, t\right)=p(t), \nabla S(q, 0)=p(0) .
$$

For a generic $S(q, 0)$ only one such trajectory exists.

The 'local' solution of the continuity equation (3) is also straightforward. At a given starting point $q_{0}$ the momentum is given by $p_{0}=\nabla S_{0}\left(q_{0}\right)$, and the amplitude of the wave function is $\varphi_{0}(q)$. After time $t$ the coordinate $q_{0}$ evolves to $q(t)$ and $p_{0}$ to $p(t)$ according to the full Hamiltonian flow. The new amplitude can be derived from the probability conservation as follows: Take an infinitesimal initial $d$ dimensional directed volume $V\left(q_{0}\right)$ around $q_{0}$ in the configuration space. The points of this volume evolve to the infinitesimal directed volume $V(q(t))$ around $q(t)$ according to the Hamiltonian flow. The value of the amplitude changes according to

$$
\varphi(q(t))= \pm\left(\frac{V(q(t))}{V(q(0))}\right)^{-1 / 2} \varphi_{0}\left(q_{0}\right)
$$

where the sign \pm 1 reflects the ambiguity of the transformation from the density $\varrho$ to $\varphi$. Careful analysis shows that the minus sign refers to the case where the orientation of the final volume is the opposite the initial one. The ratio of volumes is independent of the way we specify the initial infinitesimal volume. To understand which quantities determine the volume ratio, we specify an initial directed parallelepiped around $q_{0}$ with edges given by $d$ independent infinitesimal vectors $\delta \mathbf{q}_{1}, \delta \mathbf{q}_{2}, \ldots \delta \mathbf{q}_{d}$. After time $t$ these vectors are transformed into $\delta \mathbf{q}_{1}^{\prime}, \delta \mathbf{q}_{2}^{\prime}{ }_{2}, \ldots \delta \mathbf{q}_{d}^{\prime}$ by the flow. The initial point $\left(q_{0}, p_{0}\right)$ and the set of initial vectors do not specify uniquely the image vectors. This is a consequence of the fact that the infinitesimal vectors are transformed by the full Jacobian matrix of the Hamilton flow, and we have not specified the infinitesimal momentum vectors around $p_{0}$ corresponding to these vectors. The initial function $S_{0}(q)$ determines a set $\delta \mathbf{p}_{1}, \delta \mathbf{p}_{2}, \ldots \delta \mathbf{p}_{d}$ of vectors through the second derivative matrix:

$$
\delta \mathbf{p}=\mathbf{M} \delta \mathbf{q}, \quad \mathbf{M}_{j i}=\frac{\partial^{2} S_{0}(q)}{\partial q_{j} \partial q_{i}},
$$

which we shall call the curvature matrix. The initial curvature matrix $\mathbf{M}_{0}$ is an important initial condition, and we are not able to compute the image of the 
volume without it. The vector $\left(\delta \mathbf{q}_{i}, \delta \mathbf{p}_{i}\right)$ is transformed by the Jacobi matrix

$$
\begin{aligned}
\delta \mathbf{q}^{\prime}{ }_{i} & =\mathbf{J}_{q q} \delta \mathbf{q}_{i}+\mathbf{J}_{q p} \delta \mathbf{p}_{i}, \\
\delta \mathbf{p}^{\prime}{ }_{i} & =\mathbf{J}_{p q} \delta \mathbf{q}_{i}+\mathbf{J}_{p p} \delta \mathbf{p}_{i},
\end{aligned}
$$

where the subscripts $q$ and $p$ denote the corresponding $[d \times d]$ submatrices of the full $[2 d \times 2 d]$ Jacobian. The Jacobian is determined by the initial condition $\left(q_{0}, p_{0}\right)$ and can be computed as a time ordered integral along the phase space trajectory

$$
\mathbf{J}(q, p, t)=\mathrm{T} \exp \left\{\int_{0}^{t} d \tau \mathbf{D}^{2} H(q(\tau), p(\tau))\right\},
$$

where $\mathbf{D}^{2} H(q, p)$ denotes the second symplectic derivative matrix of the Hamiltonian and $\mathrm{T}$ is the time ordering operator. The curvature matrix of the function $S(q, t)$ around $q(t)$ defines a (8) type relation between the infinitesimal vectors

$$
\delta \mathbf{p}^{\prime}=\mathbf{M}^{\prime} \delta \mathbf{q}^{\prime}, \quad \mathbf{M}_{j i}^{\prime}=\frac{\partial^{2} S\left(q^{\prime}, t\right)}{\partial q_{j} \partial q_{i}} .
$$

Using (8) and (13), we can eliminate the vectors $\delta \mathbf{p}_{i}$ and $\delta \mathbf{p}^{\prime}{ }_{i}$ and can get relations between the initial and final infinitesimal configuration vectors and the curvature matrices

$$
\begin{aligned}
\delta \mathbf{q}^{\prime} & =\left(\mathbf{J}_{q q}+\mathbf{J}_{q p} \mathbf{M}\right) \delta \mathbf{q}, \\
\mathbf{M}^{\prime} & =\left(\mathbf{J}_{p q}+\mathbf{J}_{p p} \mathbf{M}\right)\left(\mathbf{J}_{q q}+\mathbf{J}_{q p} \mathbf{M}\right)^{-1} .
\end{aligned}
$$

From the first relation (14) we can read off the volume ratio:

$$
\frac{V\left(q^{\prime}\right)}{V\left(q_{0}\right)}=\operatorname{det}\left(\mathbf{J}_{q q}+\mathbf{J}_{q p} \mathbf{M}\right)
$$

The second relation (15) is a recursion relation for $\mathbf{M}$ and can be considered as the matrix generalization of the usual fractional rational transformation. From (15) we can derive a differential equation for $\mathbf{M}(t)$, if we substitute the elements of the infinitesimal Jacobi matrix. This differential equation

$$
\dot{\mathbf{M}}=-\left(\frac{\partial^{2} H}{\partial q \partial q}+\mathbf{M} \frac{\partial^{2} H}{\partial p \partial q}+\frac{\partial^{2} H}{\partial q \partial p} \mathbf{M}+\mathbf{M} \frac{\partial^{2} H}{\partial p \partial p} \mathbf{M}\right),
$$

is a driven one since the second partial derivatives of the Hamilton function should be computed along the phase space trajectory. If we solve this differential 
equation along the phase space trajectory the volume ratio can be written as a time ordered integral along the phase space and $\mathbf{M}(t)$ trajectory

$$
\frac{V\left(q^{\prime}\right)}{V\left(q_{0}\right)}=\mathrm{T} \exp \left\{\int_{0}^{t} \operatorname{Tr}\left[\frac{\partial^{2} H}{\partial p \partial q}+\frac{\partial^{2} H}{\partial p \partial p} \mathbf{M}\right] d \tau\right\}
$$

The square root of the volume ratio is also a time ordered integral:

$$
\left(\frac{V\left(q^{\prime}\right)}{V\left(q_{0}\right)}\right)^{-1 / 2}=\mathrm{T} \exp \left\{-\frac{1}{2} \int_{0}^{t} \operatorname{Tr}\left[\frac{\partial^{2} H}{\partial p \partial q}+\frac{\partial^{2} H}{\partial p \partial p} \mathbf{M}\right] d \tau\right\} .
$$

The computation of this expression requires some care when the solution of the differential equation (17) is singular. Close to a singularity, where

$$
\mathbf{M}\left(t \rightarrow t^{c}\right)=\infty
$$

we can neglect the non-leading terms from (17) and use the solution of

$$
\dot{\mathbf{M}}=-\mathbf{M} \frac{\partial^{2} H}{\partial p \partial p} \mathbf{M}
$$

The second derivative matrix can be decomposed into combinations of dyads and their eigenvalues in the usual way

$$
\frac{\partial^{2} H}{\partial p \partial p}=\sum_{i=1}^{d} \lambda_{i} \mathbf{D}_{i}, \quad \mathbf{D}_{i} \mathbf{D}_{j}=\delta_{i j} \mathbf{D}_{j} .
$$

The solution close to the singularity can be a linear combination of some of these dyads corresponding to singular directions $l$ :

$$
\mathbf{M}(t)=\frac{1}{t-t^{c}} \sum_{l=1}^{R} \frac{1}{\lambda_{l}} \mathbf{D}_{l},
$$

where $R$ is the number of singular directions. The time ordered integral close to the singularity is dominated by

$$
\left(\frac{V\left(q\left(t_{+0}^{c}\right)\right)}{V\left(q\left(t_{-0}^{c}\right)\right)}\right)^{-1 / 2}=\exp \left(-\frac{1}{2} \int_{t_{c}-0}^{t_{c}+0} \frac{R}{\tau-t_{c}} d \tau\right) .
$$

This integral can be computed by adding infinitesimal imaginary value $i \epsilon$ to $t^{c}$ and taking the $\epsilon \rightarrow 0$ limit

$$
\left(\frac{V\left(q\left(t_{+0}^{c}\right)\right)}{V\left(q\left(t_{-0}^{c}\right)\right)}\right)^{-1 / 2}=\exp (i \pi(R / 2))
$$


Between two singular points the time ordered integral is positive and gives the absolute value of the volume ratio. Notice that $R$ counts the number of rank reductions of the matrix $\mathbf{M}$ along the classical path, and it is also a function on the initial condition $\mathbf{M}_{0}$.

Now we have everything needed to describe the time evolution of a quasiclassical wave function. The wave function at time $t$ is now

$$
\psi\left(q^{\prime}, t\right)=\varphi\left(q^{\prime}, t\right) e^{i S\left(q^{\prime}, t\right) / \hbar}= \pm\left(\frac{V\left(q^{\prime}\right)}{V\left(q_{0}\right)}\right)^{-1 / 2} e^{i \int_{0}^{t} L d \tau / \hbar} \varphi_{0}\left(q_{0}\right) e^{i S_{0}\left(q_{0}\right) / \hbar}
$$

where $q_{0}$ is the starting point of a classical trajectory with initial momentum $\nabla S_{0}\left(q_{0}\right)$ which ends up in $q$ after time $t$ with momentum $\nabla S(q, t)$, and the volume ratio is determined by the curvature matrix $\mathbf{M}=\partial_{i} \partial_{j} S(q, 0)$.

\section{Time evolution à la Maslov}

At this point it is possible to express the volume ratio and the momentum with the second and first derivatives of the minimal action between $q^{\prime}$ and $q$. In this way we recover the usual Van Vleck propagator 13. The spirit of equation (25) is that the wave amplitude $\varphi$ at time $t$ and at coordinate $q^{\prime}$ is determined by the amplitude at $t=0$ at coordinate $q$. In calculations involving the Van Vleck operator kernel this nice property is lost, and we have to compute lots of trajectories to compute the volume ratio and we have to know the whole initial wave function too. However we have a better option. We can keep track of the variables $p$ and $\mathbf{M}$ along only one trajectory and compute (6) and the volume ratio (19). This means that the evolution takes place on the extended $(q, p, \mathbf{M})$

space. We can introduce classical density functions $\tilde{\psi}$ defined on this space. The wave function then corresponds to the special function

$$
\tilde{\psi}(q, p, \mathbf{M}, t)=\psi(q, t) \delta(p-\nabla S(q, t)) \delta\left(\mathbf{M}-\frac{\partial^{2} S(q, t)}{\partial q_{j} \partial q_{i}}\right) .
$$

The evolution of a general classical density function on the extended space according to (25) can be rewritten in terms of a classical transfer operator

$$
\tilde{\psi}\left(q^{\prime}, p^{\prime}, \mathbf{M}^{\prime}, t\right)=\int d q d p d \mathbf{M} \mathcal{L}\left(q^{\prime}, p^{\prime}, \mathbf{M}^{\prime}, t \mid q, p, \mathbf{M}, 0\right) \tilde{\psi}(q, p, \mathbf{M}, 0)
$$


with the kernel

$e^{i \pi \nu+\int_{0}^{t} d \tau \frac{i L}{\hbar}+\frac{1}{2} \operatorname{Tr}\left\{\frac{\partial^{2} H}{\partial p \partial q}+\frac{\partial^{2} H}{\partial p \partial p} \mathbf{M}\right\}} \delta\left(q^{\prime}-q^{t}(q, p)\right) \delta\left(p^{\prime}-p^{t}(q, p)\right) \delta\left(\mathbf{M}^{\prime}-\mathbf{M}^{t}(q, p, \mathbf{M})\right)$,

where $q^{t}(q, p), p^{t}(q, p)$ and $\mathbf{M}^{t}(q, p, \mathbf{M})$ denote the evolution of $q, p$ and $\mathbf{M}$ from the initial coordinates $q, p=\nabla S_{0}(q)$ and $\mathbf{M}=\partial_{i} \partial_{j} S_{0}(q)$ during the time $t$, and $\nu=N+R / 2$. The time ordered integrals should be computed along the full trajectory, and also the number of rank reductions $R$ and the number of orientation changes $N$. The Dirac deltas assure that the operator connects coordinates, which are connected by the classical dynamics, and give the correct amplitude. This operator can evolve densities, which are not of the form (26), and therefore we can expect that only a part of its spectrum has relevance to semiclassics, but all the semiclassical eigenvalues will be contained in its spectra.

\section{Entire Fredholm determinants}

The spectral or Fredholm determinant of the operator (28) can be defined by the identity

$$
\operatorname{det}(1-\mathcal{L})=\exp \left(-\sum_{n=1}^{\infty} \frac{1}{n} \operatorname{Tr} \mathcal{L}^{n}\right),
$$

where the traces of the powers of the operator are

$$
\operatorname{Tr} \mathcal{L}^{n}=\int d q d p d \mathbf{M} \mathcal{L}^{n}(q, p, \mathbf{M} \mid q, p, \mathbf{M}) .
$$

The zeroes of the Fredholm determinant on the complex energy plane yield the eigenvalues of the operator. The simplest application of (28) is to 3-dimensional hyperbolic Hamiltonian flows. In this case the spectral or Fredholm determinant of the operator (28) is given by $[8$

$$
\begin{aligned}
\operatorname{det}(\mathbf{1}-\mathcal{L}) & =\exp \left(-\sum_{p, r} \frac{1}{r\left|\Lambda_{p}^{r}\right|} \frac{e^{i r S_{p}(E)+i \nu_{p} \pi}}{\left(1-1 / \Lambda_{p}^{r}\right)^{2}} \Delta_{p, r}\right) \\
\Delta_{p, r} & =\frac{\left|\Lambda_{p}^{r}\right|^{1 / 2}}{1-1 / \Lambda_{p}^{2 r}}+\frac{\left|\Lambda_{p}^{r}\right|^{-5 / 2}}{1-1 / \Lambda_{p}^{2 r}}
\end{aligned}
$$

where $p$ is the index of primitive periodic orbits, $\Lambda_{p}$ is the corresponding eigenvalue of the stability matrix, and $r$ is the repetition number. The practical 
advantage of (31) over the more familiar Gutzwiller-Voros and Ruelle type zeta functions was demonstrated by detailed numerical studies [7] of the related quantum Fredholm determinant 14. It can be shown 15] that the Fredholm determinant obtained by keeping only one of the terms in the sum in (31) is an entire function in the whole energy plane. This enables us to show that the GutzwillerVoros spectral determinant for Axiom A flows is meromorphic in the complex $E$ plane, as it can be written as a ratio of entire functions [8]. The non-physical eigenvalues of 28 can be removed.

\section{Conclusions}

In conclusion, we have constructed a classical evolution operator for semiclassical quantization based on Maslov's quasi-classical quantization method, and derived a new determinant for periodic orbit quantization of chaotic dynamical systems. The main virtue of the determinant (31) is that the theorem of H.H. Rugh [6], applicable to multiplicative evolution operators such as (28), implies that this determinant should be entire for Axiom A flows, i.e. free of poles in the entire complex energy plane. Our numerical tests of the three disk system also support the above claims 15 .

\section{Acknowledgments}

The author thanks useful comments from P. Cvitanović, P. E. Rosenqvist, S. Creagh, P. Dimon and A. Wirzba, and is grateful to the Széchenyi Foundation, the George Soros Foundation, OTKA F4286 and the organizers for support, and to the Center for Chaos and Turbulence Studies, Niels Bohr Institute for hospitality.

\section{References}

[1] M. C. Gutzwiller, Chaos in Classical Mechanics (Springer, New York 1990)

[2] A. Voros, J. Phys. A 21, 685 (1988)

[3] B. Eckhard and G. Russberg, Phys. Rev. E 47, 1578 (1993) 
[4] D. Ruelle, Statistical Mechanics, Thermodynamical Formalizm (AddisonWesley, Reading MA, 1987)

[5] P. Szépfalusy, T. Tél, A. Csordás and Z. Kovács, Phys. Rev. A 36, 3525 (1987)

[6] H. H. Rugh, Nonlinearity 5, 1237 (1992) and H. H. Rugh, Thesis (1993)

[7] P. Cvitanović, P. E. Rosenqvist, H. H. Rugh and G. Vattay, Scattering Theory - special issue, CHAOS (1993)

[8] P. Cvitanović, G. Vattay, To be published.

[9] V. I. Arnold, Mathematical Methods of Classical Mechanics, Graduate Texts in Mathematics 60, Springer-Verlag, New York 1987.

[10] V. P. Maslov and M. V. Fedoriuk, Semi-Classical Approximation in Quantum Mechanics (Redel, Boston 1981)

[11] E. J. Heller, S. Tomsovic and A. Sepúlveda CHAOS 2, Periodic Orbit Theory - special issue, 105, 1992

[12] V. I. Arnold, Geometrical Methods in the Theory of Ordinary Differential Equations, SpringerVerlag, New York 1983.

[13] R. G. Littlejohn, Preprint 1991.

[14] P. Cvitanović and P.E. Rosenqvist, Conference Proceedings, 41, ed.: G. F. Dell'Antonio, S. Fantoni and V. R. Manfredi (Italian Physical Society, Bologna 1993)

[15] G. Vattay, To be published. 